\title{
THE KOLMOGOROFF PRINCIPLE FOR THE LEBESGUE AREA
}

\author{
R. G. HELSEL AND E. J. MICKLE
}

1. Introduction. Kolmogoroff [3] $]^{1}$ has considered an axiomatic development of measure theory for analytic sets in a metric space. One of his axioms is the following: If the set $E^{*}$ is the image of a set $E$ under a Lipschitzian transformation with Lipschitz constant equal to or less than one, then the measure of $E^{*}$ should not exceed the measure of $E$. As stated, the principle has meaning only for point sets, but we shall be concerned with the area (or two-dimensional measure) of Fréchet surfaces in Euclidean 3-space; so we first reformulate the Kolmogoroff principle as follows: If $T^{*}$ and $T$ are two continuous transformations from the unit disc $D: u^{2}+v^{2} \leqq 1$ into Euclidean 3-space which satisfy the distance inequality $\mid T^{*}\left(p_{1}\right)$ $-T^{*}\left(p_{2}\right)|\leqq| T\left(p_{1}\right)-T\left(p_{2}\right) \mid$ for every pair of points $p_{1}, p_{2} \in D$, then the area of the Fréchet surface determined by $T^{*}$ should not exceed the area of the one determined by $T$. The purpose of this paper is to show that the Kolmogoroff principle, as reformulated above for Fréchet surfaces, is satisfied by the Lebesgue area.

We first discuss the case in which both the Fréchet surfaces are represented by Lipschitzian transformations. Since the Lebesgue area is given by the usual double integral formula in this case (see Radó [5]), an elementary inequality suffices to establish the desired relation between the areas. In an independent study of a related question, Reichelderfer [6] has obtained a result which can be shown to be equivalent. The general case, in which the transformations representing the surfaces are merely assumed to be continuous, depends upon the extension of a Lipschitzian vector function, defined originally on a bounded and closed set, to an open neighborhood of the set. We are indebted to Professor S. Eilenberg for calling our attention to the fact that such an extension has already been established by Kirszbraun [2].

2. The Lipschitz case. To begin, let us establish the elementary inequality mentioned in the introduction.

Lemma 1. If $E, F, G$ and $E^{*}, F^{*}, G^{*}$ are constants such that

Presented to the Society, September 5, 1947; received by the editors June 9, 1947.

${ }_{1}^{1}$ Numbers in brackets refer to the bibliography at the end of the paper. 
$E \cos ^{2} \theta+2 F \cos \theta \sin \theta+G \sin ^{2} \theta \geqq E^{*} \cos ^{2} \theta+2 F^{*} \cos \theta \sin \theta+G^{*} \sin ^{2} \theta$ $\geqq 0$ for $0 \leqq \theta \leqq 2 \pi$, then $E G-F^{2} \geqq E^{*} G^{*}-F^{* 2}$.

Proof. Since the quadratic form $E^{*} \cos ^{2} \theta+2 F^{*} \cos \theta \sin \theta+G^{*} \sin ^{2} \theta$ is positive, we have

$$
E^{*} G^{*}-F^{* 2} \geqq 0 .
$$

The quadratic form $\left(E-E^{*}\right) \cos ^{2} \theta+2\left(F-F^{*}\right) \cos \theta \sin \theta+\left(G-G^{*}\right)$ $\sin ^{2} \theta$ is also positive; so

$$
\left(E-E^{*}\right)\left(G-G^{*}\right) \geqq\left(F-F^{*}\right)^{2},
$$

which may be written in the form

$$
\begin{aligned}
E G-F^{2} \geqq & E^{*} G^{*}-F^{* 2}+G^{*}\left(E-E^{*}\right) \\
& -2 F^{*}\left(F-F^{*}\right)+E^{*}\left(G-G^{*}\right) .
\end{aligned}
$$

Clearly the desired inequality will follow from (3) if we can show that

$$
G^{*}\left(E-E^{*}\right)-2 F^{*}\left(F-F^{*}\right)+E^{*}\left(G-G^{*}\right) \geqq 0 .
$$

The numbers $G^{*}\left(E-E^{*}\right)$ and $E^{*}\left(G-G^{*}\right)$ are non-negative; hence $G^{*}\left(E-E^{*}\right)+E^{*}\left(G-G^{*}\right) \geqq 2\left[G^{*}\left(E-E^{*}\right) E^{*}\left(G-G^{*}\right)\right]^{1 / 2}$. But multiplication of (1) and (2) yields the inequality $\left[G^{*}\left(E-E^{*}\right) E^{*}\left(G-G^{*}\right)\right]^{1 / 2}$ $\geqq\left|F^{*}\left(F-F^{*}\right)\right|$ and $(4)$ now follows.

In the sequel it will be convenient to use $x$ and $y$ to represent vectors in Euclidean 3-space with components $\left(x_{1}, x_{2}, x_{3}\right)$ and $\left(y_{1}, y_{2}, y_{3}\right)$ respectively. The $x_{1} x_{2} x_{3}$ and $y_{1} y_{2} y_{3}$ spaces may or may not coincide. If the components of $x$ are functions of the parameters $u$ and $v$, we shall indicate the fact by the notation $\mathfrak{x}(u, v)$. Then $\mathfrak{x}_{u}(u, v)$ and $\mathfrak{x}_{v}(u, v)$ will denote vector functions whose components are obtained from those of $\mathfrak{x}(u, v)$ by taking the indicated partial derivative. We shall also use the conventional notations:

$$
E=\mathfrak{x}_{u}^{2}, \quad F=\mathfrak{x}_{u} \cdot \mathfrak{x}_{v}, \quad G=\mathfrak{x}_{v}^{2}, \quad W=\left(E G-F^{2}\right)^{1 / 2} .
$$

Lemma 2. If $\mathfrak{x}(u, v)$ is a Lipschitzian vector function on $D: u^{2}+v^{2} \leqq 1$, then, for a.e. $(u, v) \in D$,

$$
\begin{aligned}
\left|\frac{\mathfrak{x}(u+r \cos \theta, v+r \sin \theta)-\mathfrak{x}(u, v)}{r}\right| & \underset{r \rightarrow+0}{\longrightarrow}\left|\mathfrak{x}_{u}(u, v) \cos \theta+\mathfrak{x}_{v}(u, v) \sin \theta\right|
\end{aligned}
$$

for $0 \leqq \theta \leqq 2 \pi$.

Proof. See Rademacher [4] for the case of a single-valued function. 
The extension to a vector function is immediate.

THEOREM 1. If $T^{*}: \mathfrak{x}=\mathfrak{r}^{*}(u, v)$ and $T: \mathfrak{x}=\mathfrak{x}(u, v)$ are Lipschitzian transformations defined on $D: u^{2}+v^{2} \leqq 1$ such that $\left|\mathfrak{x}^{*}\left(u_{1}, v_{1}\right)-\mathfrak{r}^{*}\left(u_{2}, v_{2}\right)\right|$ $\leqq\left|\mathfrak{x}\left(u_{1}, v_{1}\right)-\mathfrak{x}\left(u_{2}, v_{2}\right)\right|$ for every pair of points $\left(u_{1}, v_{1}\right),\left(u_{2}, v_{2}\right) \in D$, then $A\left(T^{*}\right) \leqq A(T)$ (where $A\left(T^{*}\right)$ and $A(T)$ are the Lebesgue areas of the Fréchet surfaces determined by $T^{*}$ and $T$ ).

Proof. Since $\left|\mathfrak{r}^{*}(u+r \cos \theta, v+r \sin \theta)-\mathfrak{r}^{*}(u, v)\right| \leqq \mid \mathfrak{x}(u+r \cos \theta$, $v+r \sin \theta)-\mathfrak{x}(u, v) \mid$, providing $(u, v),(u+r \cos \theta, v+r \sin \theta) \in D$, it follows from Lemma 2 that, a.e. in $D$,

$$
\left|\mathfrak{x}_{u}^{*}(u, v) \cos \theta+\mathfrak{x}_{v}^{*}(u, v) \sin \theta\right| \leqq\left|\mathfrak{x}_{u}(u, v) \cos \theta+\mathfrak{x}_{v}(u, v) \sin \theta\right|
$$

for $0 \leqq \theta \leqq 2 \pi$. Squaring both sides of this inequality, we have, a.e. in $D, 0 \leqq E^{*} \cos ^{2} \theta+2 F^{*} \cos \theta \sin \theta+G^{*} \sin ^{2} \theta \leqq E \cos ^{2} \theta+2 F \cos \theta \sin \theta$ $+G \sin ^{2} \theta$ for $0 \leqq \theta \leqq 2 \pi$. Thus, by Lemma 1 , a.e. in $D, W^{* 2}=E^{*} G^{*}$ $-F^{* 2} \leqq E G-F^{2}=W^{2}$. Since $W^{*}$ and $W$ are non-negative, it follows that $W^{*} \leqq W$ a.e. in $D$, and integration yields the desired inequality $A\left(T^{*}\right)=\iint_{D} W^{*} d u d v \leqq \iint_{D} W d u d v=A(T)$.

3. The general case. To establish the Kolmogoroff principle for the Lebesgue area in the general case, we shall need the following result concerning the extension of the range of definition of a Lipschitzian vector function.

Lemma 3. If $L: \mathfrak{y}=\mathfrak{y}\left(x_{1}, x_{2}, x_{3}\right)$ is a Lipschitzian transformation defined on a bounded set $B$ in $x_{1} x_{2} x_{3}$-space, then $L$ can be defined on the complement of $B$ in such a way that the extended transformation is Lipschitzian on the whole space with the same Lipschitz constant.

Proof. See Kirszbraun [2].

LEMMA 4. If $T^{*}: \mathfrak{y}=\mathfrak{y}^{*}(u, v)$ and $T: \mathfrak{x}=\mathfrak{x}(u, v)$ are any two bounded transformations defined on $D: u^{2}+v^{2} \leqq 1$ such that $\left|\mathfrak{y}^{*}\left(u_{1}, v_{1}\right)-\mathfrak{y}^{*}\left(u_{2}, v_{2}\right)\right|$ $\leqq\left|\mathfrak{x}\left(u_{1}, v_{1}\right)-\mathfrak{x}\left(u_{2}, v_{2}\right)\right|$ for every pair of points $\left(u_{1}, v_{1}\right),\left(u_{2}, v_{2}\right) \in D$, then there exists a Lipschitzian transformation $L: \mathfrak{y}=\mathfrak{y}\left(x_{1}, x_{2}, x_{3}\right)$, with Lipschitz constant equal to one, which is defined on all of $x_{1} x_{2} x_{3}$-space and is such that $T^{*}=L T$ on $D$.

Proof. Let $p$ be any point in $T(D)$. Consider the inverse set $T^{-1}(p)$. It is clear, since $\left|\mathfrak{y}^{*}\left(u_{1}, v_{1}\right)-\mathfrak{h}^{*}\left(u_{2}, v_{2}\right)\right| \leqq\left|\mathfrak{x}\left(u_{1}, v_{1}\right)-\mathfrak{x}\left(u_{2}, v_{2}\right)\right|$ for every pair of points $\left(u_{1}, v_{1}\right),\left(u_{2}, v_{2}\right) \in D$, that under $T^{*}$ every point in $T^{-1}(p)$ must have one and the same image $p^{*}$. Thus $T^{*} T^{-1}$ is a Lipschitzian transformation from $T(D)$ to $T^{*}(D)$, with Lipschitz constant equal to one, such that $\left(T^{*} T^{-1}\right) T=T^{*}$ on $D$. By Lemma 3 , there exists a 
transformation $L$ (the extension of $T^{*} T^{-1}$ ) satisfying the requirements of the lemma.

THEOREM 2. If $T^{*}: \mathfrak{y}=\mathfrak{y}^{*}(u, v)$ and $T: \mathfrak{x}=\mathfrak{x}(u, v)$ are continuous transformations defined on $D: u^{2}+v^{2} \leqq 1$ such that $\left|\mathfrak{y}^{*}\left(u_{1}, v_{1}\right)-\mathfrak{y}^{*}\left(u_{2}, v_{2}\right)\right|$ $\leqq\left|\mathfrak{x}\left(u_{1}, v_{1}\right)-\mathfrak{x}\left(u_{2}, v_{2}\right)\right|$ for every pair of points $\left(u_{1}, v_{1}\right),\left(u_{2}, v_{2}\right) \in D$, then $A\left(T^{*}\right) \leqq A(T)$.

Proof. Let $L$ be the Lipschitzian transformation satisfying the requirements of Lemma 4 and let $T_{n}$ be a sequence of Lipschitzian transformations defined on $D$ and such that $T_{n} \rightarrow T$ uniformly on $D$ and $A\left(T_{n}\right) \rightarrow A(T)$ (see Youngs [7], Helsel [1]). Define $T_{n}{ }^{*}=L T_{n}$ on $D$. Then $T_{n}^{*} \rightarrow T^{*}$ uniformly on $D$ and the Lipschitzian transformations $T_{n}^{*}$ and $T_{n}$ satisfy the hypotheses of Theorem 1 . Therefore, $A\left(T_{n}^{*}\right) \leqq A\left(T_{n}\right)$ and, by the lower semi-continuity of the Lebesgue area, $A\left(T^{*}\right) \leqq \lim \inf A\left(T_{n}^{*}\right) \leqq \lim A\left(T_{n}\right)=A(T)$.

\section{BIBLIOGRAPHY}

1. R. G. Helsel, $A$ theorem on surface area, Trans. Amer. Math. Soc. vol. 61 (1947) pp. 443-453.

2. M. D. Kirszbraun, Über die zusammenziehende und Lipschitzsche Transformationen, Fund. Math. vol. 22 (1934) pp. 77-108.

3. A. Kolmogoroff, Beitrage zur Masstheorie, Math. Ann. vol. 107 (1933) pp. 351366.

4. H. Rademacher, Über partielle und totale Differenzierbarkeit von Funktionen mehrerer Variabeln und ibber die Transformation der Doppelintegrale, Math. Ann. vol. 79 (1918) pp. 340-359.

5. T. Rad6, Über das Flächenmass rektifizierbarer Flächen, Math. Ann. vol. 100 (1928) pp. 445-479.

6. P. V. Reichelderfer, The effect of a Lipschitzian transformation on the area of a continuous surface. Bull. Amer. Math. Soc. Abstract 53-5-225.

7. J. W. T. Youngs, The additivity of the Lebesgue area, Bull. Amer. Math. Soc. vol. 49 (1943) pp. 779-784.

The Ohio State University 